\title{
Article
}

\section{Effects of the Molar Ratio of Counter-Ions on Flow Characteristics of Surfactant Solutions Sweeping Cavities}

\author{
Hideki SATO", Hiroshi SUZUKI"*,", Ruri HidEMA ${ }^{* * *}$, and Yoshiyuki KomOdA ${ }^{* *}$ \\ *Department of Chemical Science and Engineering, Kobe University \\ 1-1 Rokkodai-cho, Nada-ku, Kobe-shi, Hyogo 657-8501, Japan \\ ** Complex Fluid and Thermal Engineering Research Center (COFTEC), \\ Department of Chemical Science and Engineering, Kobe University \\ 1-1 Rokkodai-cho, Nada-ku, Kobe-shi, Hyogo 657-8501, Japan \\ *** Complex Fluid and Thermal Engineering Research Center (COFTEC), \\ Organization of Advanced Science and Technology, Kobe University \\ 1-1 Rokkodai-cho, Nada-ku, Kobe-shi, Hyogo 657-8501, Japan \\ (Received : February 1, 2016)
}

\begin{abstract}
An experimental study has been performed in order to investigate the optimum rheological characteristics for a technique of heat transfer augmentation in a cavity between ribs mounted in parallel plates. The rheological characteristics of visco-elastic fluid were changed by controling the molar ratio of counter-ion to a cationic surfactant. Flow visualization experiments and pressure loss measurements were conducted in the range of solvent Reynolds number from 100 to 2,500 and in the range of molar ratio from 0.50 to 10 . Under this condition, the zoro-shear Reynolds number and the Weissenberg number ranged from 2.01 to 120 and from $1.94 \times 10^{-2}$ to 9.15 , respectively. From the results, flow penetration into the cavity occurred in the cases from 1.5 to 5.0 of molar ratio of counter-ions. However, the pressure loss became large in the cases when the remarkable Barus effect can be observed. On the other hand, the pressure loss became almost the same as that of water in the case when the molar ratio of counter-ions was set at 5.0. From this, it was concluded that the optimum fluid for the heat transfer augmentation in a cavity is required to have suitable elasticity and low viscosity as the molar ratio of counter-ions is set around 5.0.
\end{abstract}

Key Words: Viscoelastic fluid / Flow visualization / Cavity / Barus effect / Pressure loss

\section{INTRODUCTION}

In order to increase the heat transfer surface or compactness in a heat exchanger, rib-like heat transfer promoters are mounted on the heat transfer surfaces in some cases. However, in the cavity between such ribs, there exists a recirculating flow region where the convective heat transfer is greatly decreased as reported by Aung et al. ${ }^{1)}$ The heat transfer in such a recirculating region cannot be enhanced just by increasing the flow rate, and so heat accumulates there. Such a heat transfer reduction region is also observed between LSI chips in electric devices in which severe temperature conditions are needed. Thus, heat transfer augmentation in such recirculating regions is of importance for heat devices having complex geometries. $^{2-4)}$

In a previous study, Suzuki et al. ${ }^{5)}$ suggested a novel

$\dagger$ Tel: +81(78)8036490, Fax: +81(78)8036490, E-mail: hero@kobe-u.ac.jp technique using the Barus effect of visco-elastic fluid for heat transfer enhancement in a cavity between ribs mounted on a heat transfer surface as a heat transfer enhancement promoter. The previous study treated surfactant solution as a viscoelastic fluid and heat transfer experiments was performed in plane-symmetrically expanded cavities when the cavity height Reynolds number defined by solvent viscosity ranged from 1,400 to 4,000 . From this, it was found that the heat transfer in the upstream region of a cavity is extremely enhanced due to the flow penetration into a cavity. However, the heat transfer in the downstream region of the cavity was greatly reduced because a wide separation region was formed there. It was also reported that the Newtonian fluid flow becomes turbulent in such a high Reynolds number range, while the surfactant solution flow remains laminar due to the high viscosity at the same solvent Reynolds number. Thus, in such a high Reynolds number range, the suggested method was not concluded to be so effective for heat transfer enhancement. In order to improve 
the total heat transfer in a cavity by using the Barus effect, the effect of geometric parameters of the rib height and width and of the cavity length were experimentally investigated focusing on such separation region using a open channel in the previous study. ${ }^{6}$ For a rather low solvent Reynolds number of 100 , Suzuki et $a l^{7)}$ also discussed flow and heat transfer characteristics with the results of numerical computations. From these, it was found that the total heat transfer in a cavity was at least 2 times higher than that of water. In such a low Reynolds number region, the separation region size formed in the downstream region of the cavity was markedly decreased. Many types of compact heat changers works in such a low Reynolds number range. Thus, it was concluded that the suggested method is effective as a heat transfer enhancement method for compact heat exchangers. However, the previous study also revealed the pressure losses becomes 10 times higher than those of water even in a low Reynolds number range. ${ }^{8)}$ The numerical simulations also indicated that the high viscosity of the solution mainly contributes to the pressure loss in a flow path with cavities. From these results, it can be concluded that the visco-elastic fluid having low viscosity for pressure loss and enough elasticity for flow penetration into the cavity is suitable in order to improve the present method.

In this study, the effect of rheological characteristics of a visco-elastic fluid on flow and pressure loss characteristics have been performed in order to find the optimum rheological characteristics for the present heat transfer augmentation technique in a cavity. On the flow characteristics in a cavity, Mckinley et al. ${ }^{9)}$ discussed the vortex characteristics in the cases of Boger fluid, and concluded the size of these vortices can be correlated with Weissenberg number in a wide range of contraction ratios. Mongruel and Cloitre ${ }^{10)}$ also discussed recirculation size upstream of an axisymmetric orifice in the case of a polymer suspension. These recirculation characteristics are also important in order to prevent heat transfer reduction in the downstream region of the cavity.

A combination system of cationic surfactant (oleylbishydroxyethylmethyl ammonium chloride) with a counter-ion (sodium salicylate) has been used as a viscoelastic fluid in the previous ${ }^{5,6)}$ and present studies. Usui et $a l{ }^{10)}$ reported the effect of the counter-ion molar ratio to the surfactants on the rheological characteristics and on the drag reduction effects. According to this, the elasticity is decreased with the molar ratio of the counter-ions in the larger region than 1.5 and the zero-shear viscosity is also decreased in such a condition. Thus, it can be expected that there exists the optimum rheological condition for the present heat transfer augmentation technique using the Barus effect without pressure loss penalty. There exists no report on the flow characteristics from this point of view.

In this study, flow visualization experiments and pressure loss measurements have been performed. In the previous experiments, an open channel was used for flow visualization, which is convenient for the change the flow geometry but not useful for the pressure loss measurement. Then, a closed channel having an aspect ratio of 3.5 was used for experiments. The visco-elasticity is added to water by a cationic surfactant system with counter-ions and the rheological characteristics are controlled by the molar ratio of counter-ions to surfactants.

\section{EXPRIMENTAL PROCEDURES}

\subsection{Materials}

A cationic surfactant, oleylbishydroxyethylmethylanmonium chloride, $\left(\mathrm{C}_{18} \mathrm{H}_{35} \mathrm{~N}\left(\mathrm{C}_{2} \mathrm{H}_{4} \mathrm{OH}\right)_{2} \mathrm{CH}_{3} \mathrm{Cl}\right)$ was used for adding visco-elasticity to the fluid. The surfactants were dissolved in ion-exchanged water at a concentration of 2,000 wt-ppm. Sodium salicylate $\left(\mathrm{C}_{6} \mathrm{H}_{4}(\mathrm{OH}) \mathrm{COONa}\right)$, as a counter-ion supplier for rod-like micelle formation, was also added to the solution. The molar ratio of the counter-ions to the surfactants, $\xi[-]$, was changed from 0.50 to 10 . In such a range of the molar ratio of counter-ions, the visco-elasticity appears in the fluid.

\subsection{Rheological Characteristics}

The apparent viscosity, $\eta[\mathrm{Pa} \cdot \mathrm{s}]$, and the first normal stress difference, $N_{1}[\mathrm{~Pa}]$ of the fluids were measured by use of a shear-control rheometer with a cone-and-plate device as in the previous study ${ }^{11)}$ where the surfactant concentration was set at 500 wt-ppm. Lu et al. ${ }^{12)}$ on the relaxation time indicated the surfactant system shows a multi-mode relaxation behaviour. Suzuki et al. ${ }^{13)}$ analysed the relaxation behaviour of the surfactant system with a triple exponential Maxwell model as follows.

$$
\tau=\tau_{1} e^{-t / t_{r 1}}+\tau_{2} e^{-t / t_{r 2}}+\tau_{3} e^{-t / t_{r 3}}
$$

Here, $\tau[\mathrm{Pa}]$ is the total stress and $\tau_{1}[\mathrm{~Pa}], \tau_{2}[\mathrm{~Pa}]$ and $\tau_{3}[\mathrm{~Pa}]$ are the contributes from the respective relaxation behaviours to the total stress. $t[\mathrm{~s}]$ is time and $t_{\mathrm{r} 1}[\mathrm{~s}], t_{\mathrm{r} 2}[\mathrm{~s}]$ and $t_{\mathrm{r} 3}[\mathrm{~s}]$ are the relaxation times for the respective relaxation behaviours defined as $t_{\mathrm{r} 1}<t_{\mathrm{r} 2}<t_{\mathrm{r} 3}$. Figure 1 shows the effect of the molar ratio of counter-ions to the relaxation times obtained in the previous study. ${ }^{13)}$ As shown in the figure, there exist three relaxation times from 0.80 to 2.0 of the molar ratio of counterions. The longest relaxation disappears when $\xi$ is lower than 
0.50 or higher than 3.0. The second relaxation also disappears when $\xi$ is lower than 0.50 or higher than 5.0. Only the first relaxation remains in all cases.

\subsection{Flow Visualization and Pressure Loss Measurement}

Figure 2 shows the experimental apparatus for flow visualization experiments and pressure loss measurements. The visco-elastic fluids flow into a test duct with the height, $W[\mathrm{~m}]$, of $40 \mathrm{~mm}$ and the spanwise width of $75 \mathrm{~mm}$, made of transparent acrylic resin, by a pump from a reservoir tank. Five ribs with the height, $H[\mathrm{~m}]$, of $20 \mathrm{~mm}$ and the length, $R$ [m] of $100 \mathrm{~mm}$ were mounted on the bottom of the test section with $1,100 \mathrm{~mm}$ of the length. Each distance between ribs, which is the cavity length, $L[\mathrm{~m}]$, was set at $100 \mathrm{~mm}$. Flow visualization was performed with tracer particles of ionexchange resin with $0.10 \mathrm{~mm}$ of the diameter and $850 \mathrm{~kg} \cdot \mathrm{m}^{-3}$ of the density. A slit light with the width of $10 \mathrm{~mm}$ was inserted from the bottom of the cavity. The flow behaviour was taken by a video camera with $60 \mathrm{fps}$. The fourth cavity was tested in the present study in order to eliminate the effects of inlet flow fluctuation and of flow development from the results. The pressure difference between the inlet and the outlet of the test section was also measured by using a pressure difference transducer.

The solvent Reynolds number, $\operatorname{Re}[-]$, was defined with water viscosity (solvent viscosity), $\mu[\mathrm{Pa} \cdot \mathrm{s}]$ as follows.

$$
\operatorname{Re}=\frac{\rho U_{m} H}{\mu}
$$

Here, $\rho\left[\mathrm{kg} \cdot \mathrm{m}^{-3}\right]$ and $U_{\mathrm{m}}\left[\mathrm{m} \cdot \mathrm{s}^{-1}\right]$ are density of the fluids and the mean velocity in the narrow flow path. In this study, a low Reynolds number region where heat transfer can be expected to become higher than that in the water cases is focused on.

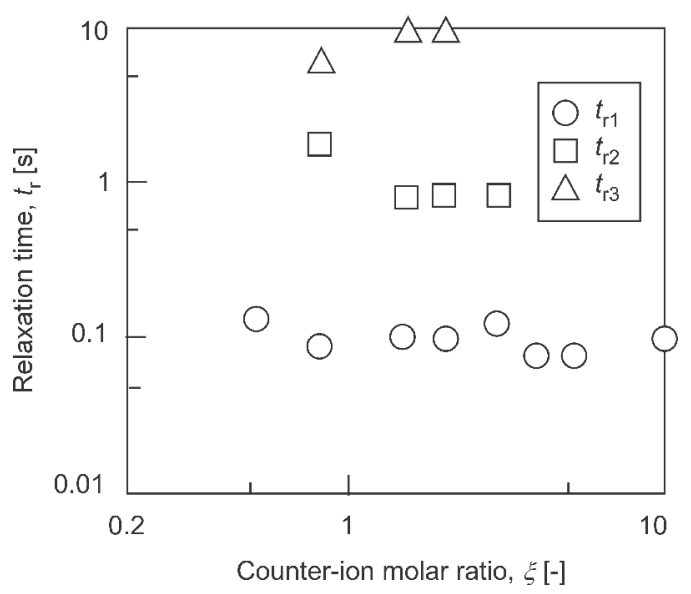

Fig. 1. Relaxation time ${ }^{13)}$.
However, the flow characteristics in the cases with cavities and flow transition behaviours in the cases of visco-elastic fluid flows have not been clarified. Then, in this study, the solvent Reynolds number was widely changed from 100 to 2,500 .

The zero-shear viscosity Reynolds number $\operatorname{Re}_{0}[-]$ and the Weissenberg number, $\mathrm{We}[-]$ were also defined as follows at the narrow path.

$$
\begin{aligned}
& R e_{0}=\frac{\rho U_{m} H}{\eta_{0}} \\
& W e=t_{r} \frac{U_{m}}{W-H}
\end{aligned}
$$

Here, $\eta_{0}[\mathrm{~Pa} \cdot \mathrm{s}]$ is the zero-shear viscosity of the surfactant solutions. $t_{\mathrm{r}}[\mathrm{s}]$ is a relaxation time. Zheng et al. ${ }^{14)}$ revealed the reconstruction from rod-like micelles to vesicles after the stress release. Suzuki et al. ${ }^{13)}$ reported the third relaxation time corresponds to this reconstruction. Thus, the first or the second relaxation time was adopted for defining the Weissenberg number as related to the elasticity of the fluids. In the case when the fluid has both the first and the second relaxation times, the second (larger) one was used as in the previous study. ${ }^{6}$ With this definition, the Weissenberg number ranged from $1.94 \times 10^{-2}$ to 9.15 in this study. The zero-shear Reynolds number ranged from 2.01 to 120 . The temperature was set at room temperature of $25^{\circ} \mathrm{C}$ during the experiments.

\section{RESULTS AND DISCUSSION}

\subsection{Rheological Characteristics}

Figures 3 and 4 show the effects of counter-ion ratio on the apparent viscosity and on the first normal stress of the fluids. From Figure 3, the apparent viscosity at $1 \mathrm{~s}^{-1}$ of shear rate is found to increase until $\xi=1.5$ and to decrease with increase of the molar ratio of the counter-ions after that. At 1.5 of the molar ratio of counter-ions, the apparent viscosity becomes

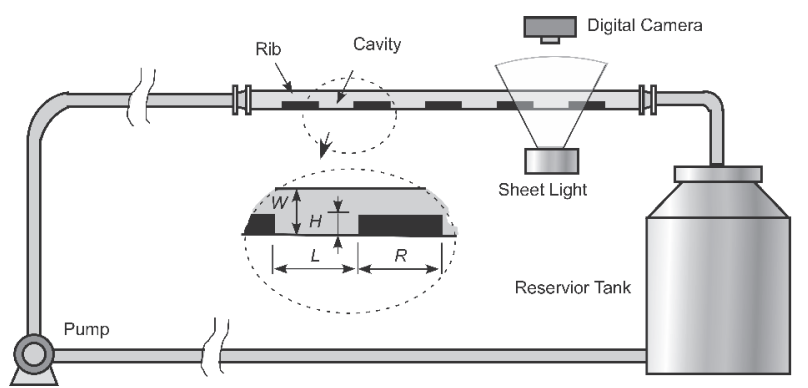

Fig. 2. Experimental Apparatus. 
80 times higher than that in the case when $\xi=10$ and shows a typical shear thinning behaviour. The effect of the molar ratio of counter-ions on the first normal stress difference corresponds to the behaviour of the viscosity as shown in Figure 4.

\subsection{Flow Visualization}

Figure 5 shows the effects of the molar ratio of counter-ions on the flow behaviour in the fourth cavity when the Reynolds number was set at 300. In the figure, a result for water is also shown. In each photo, the fluid flows from left to right and the schematic streamline separated from the top corner of the upstream rib is plotted in order to discuss the flow penetration into the cavity.

All flows shown in the figure are laminar and steady. From this figure, it is found that no flow penetration into the cavity occurs in the case of water. On the other hand, the flow separated from the top corner of the upstream rib deeply penetrates into the cavity when $\xi$ is lower. Especially in the

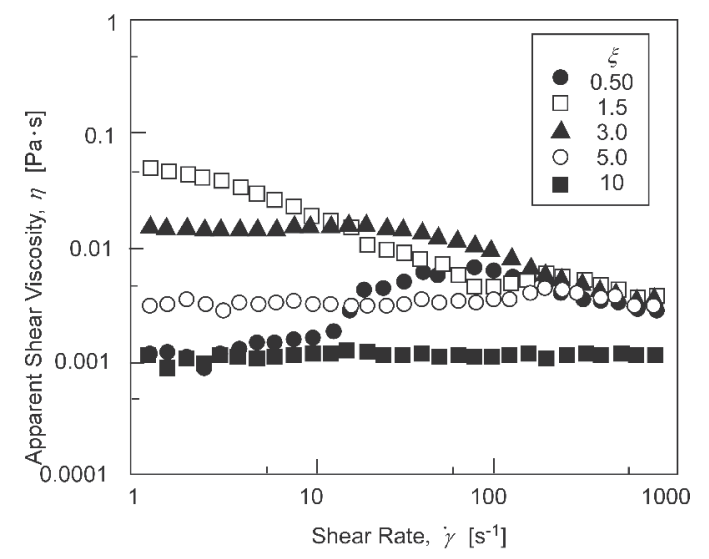

Fig. 3. Apparent viscosity.

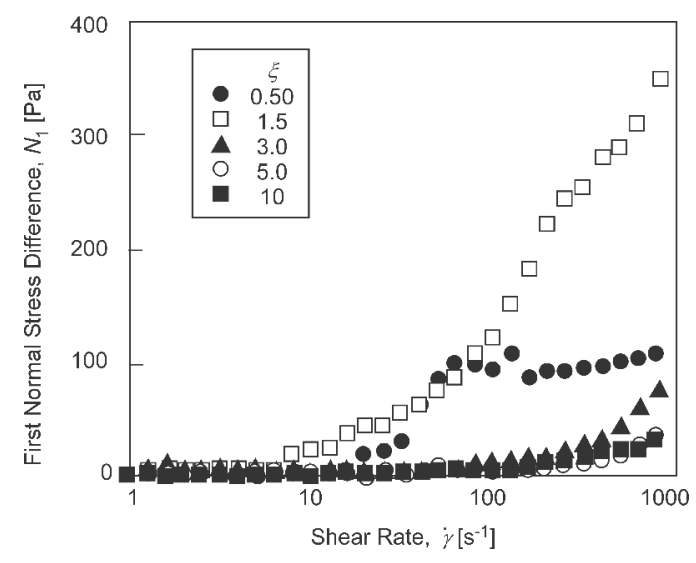

Fig. 4. First normal stress difference. case when $\xi=1.5$, the separated flow immediately reattaches onto the cavity bottom, sweeps the bottom, separates from the bottom again and moves toward the top corner of the downstream rib. The recirculating flow region forms between re-separation and the downstream rib. There, the heat transfer from the walls severely decreases. As the molar ratio of counter-ions increases from 1.5 to 5.0 , the reattachment point moves downstream and no reattachment occurs in the case when $\xi=10$.

Figure 6 shows the effects of the Reynolds number on the flow behaviour when the molar ratio of counter-ions was set at 5.0. In each photo, the schematic streamline separated from the top corner of the upstream rib is also plotted.

From the figure, it was found that the reattachment point onto the cavity bottom moves downstream with the Reynolds number. When the Reynolds number becomes higher than 1,000 , no more flow reattachment onto the cavity bottom is observed. This is because the inertia momentum becomes large. When $R e=2,500$, an unsteady vortex appears in the

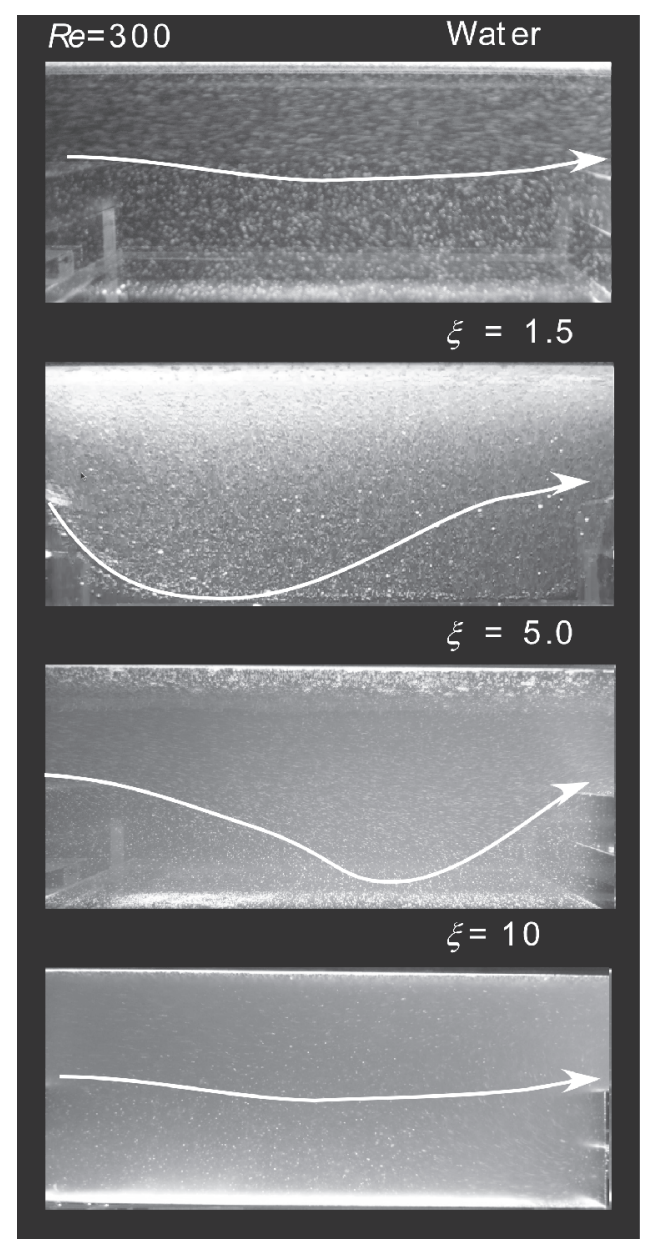

Fig. 5. Effect of the molar ratio of counter-ions on flow behaviour in the cavity. 
downstream region in the cavity and this indicates the flow in the cavity becomes turbulent.

Figures 7,8 and 9 show the reattachment length, $x_{\mathrm{r}}[\mathrm{m}]$, between the upstream rib and the reattachment point, the penetration depth, $y_{\mathrm{p}}[\mathrm{m}]$, from the top line of ribs toward the cavity bottom and the separation length, $x_{\mathrm{s}}[\mathrm{m}]$, formed by the flow re-separated from the cavity bottom, respectively. These scales were obtained from the movies. From the still images shown in Figures 5 and 6, the separation and the reattachment characteristics cannot be define precisely, but these characteristics can be clearly obtained from the movies. The accuracy of measuring these length scales is $1 \mathrm{~mm}$, which is $1 \%$ of the cavity length. In Figure 7 , the reattachment length obtained by Armaly et al. ${ }^{15)}$ experimentally for a backwardfacing Newtonian fluid flow is also plotted by a solid line. In the cases when $\xi=1.5$, the results reported in the previous study $^{16)}$ are also plotted. From Figure 8 , it is found that there exists no flow reattachment on the cavity bottom when $\xi=0.5$. Then, no data in the cases at 0.5 of the molar ratio are plotted

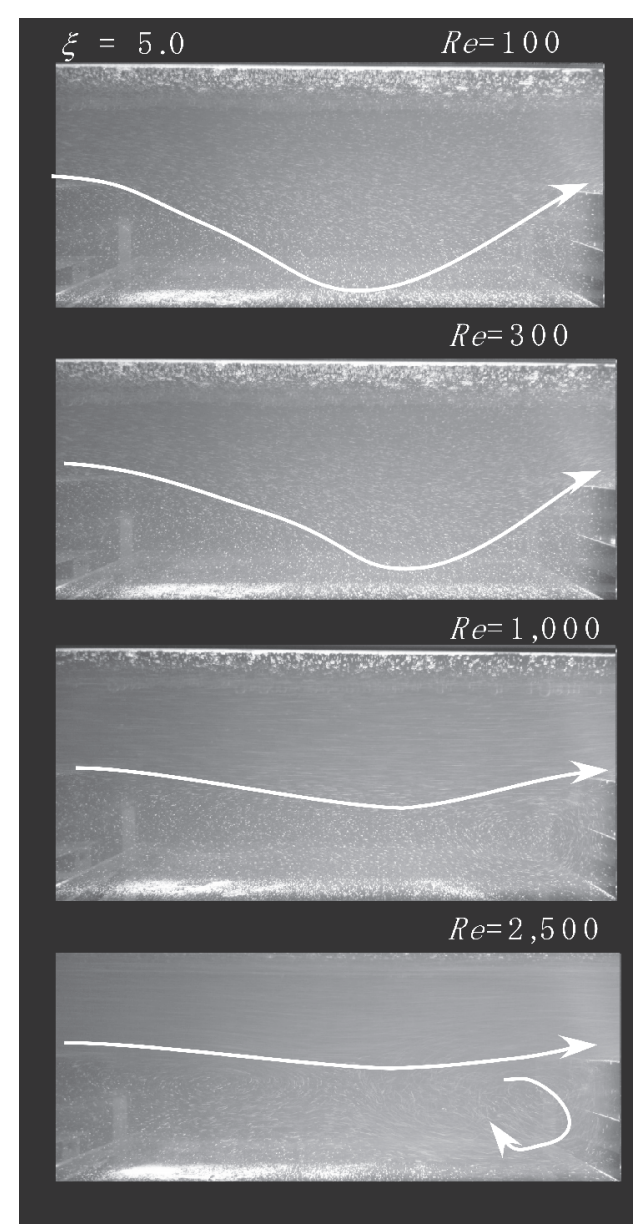

Fig. 6. Effect of the Reynolds number on flow behaviour in the cavity.

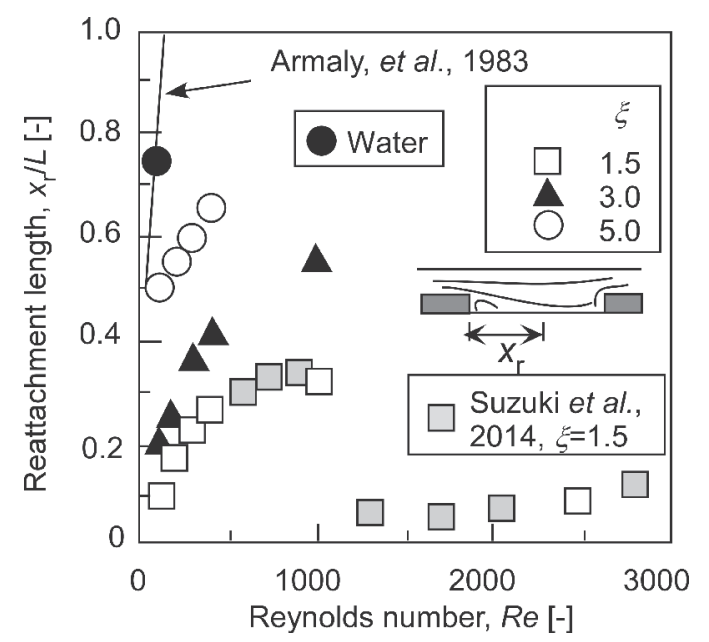

Fig. 7. Reattachment length on the solvent Reynolds number.

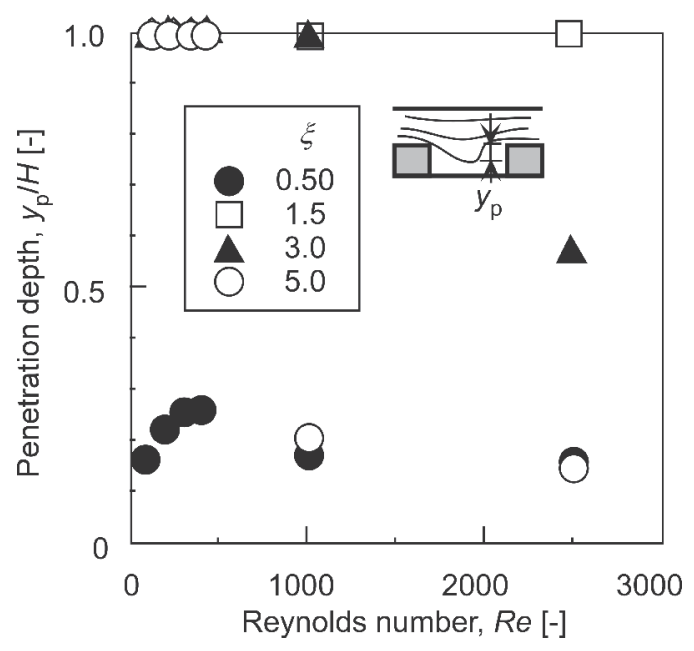

Fig. 8. Penetration depth.

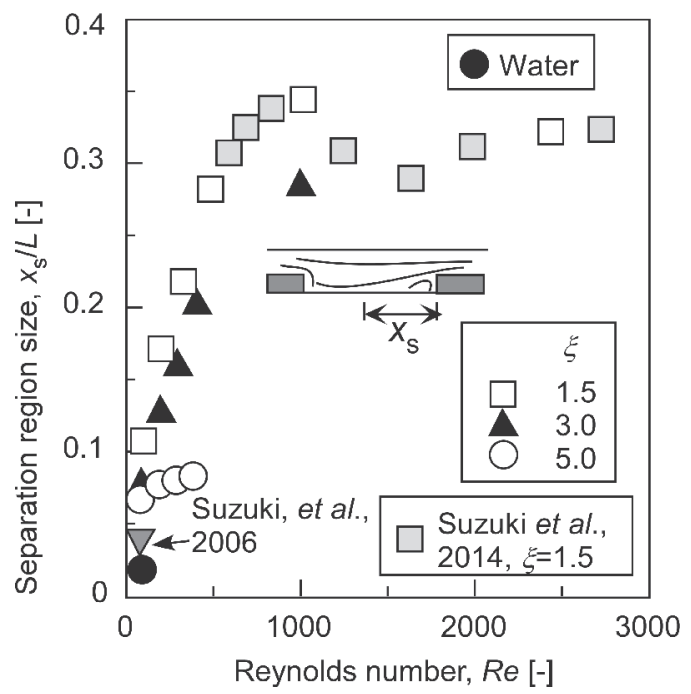

Fig. 9. Separation length. 
in Figures 7 and 9.

Chiang and Sheu ${ }^{17)}$ reported results of three-dimensional numerical computations for a backward-facing Newtonian fluid flow. They changed the aspect ratio from 2 to 100 . They revealed that the three dimensionally fluctuation of reattachment points appears from 35 to 50 of the aspect ratio when the Reynolds number was set at 400 . The present aspect ratio is set at 3.5 and then three-dimensional fluctuation can be assumed to be weak in the lower Reynolds number region than 400 .

As discussed above, the reattachment length decreases when the Reynolds number is low and the molar ratio decreases from 5.0 to 1.5. The solid line in Figure 7 shows the reattachment length becomes larger than the cavity size in the case of water when the Reynolds number is set at 200. Thus, the reattachment is observed only at 100 in the case of water. The reattachment length increases linearly in each case of the molar ratio of counter-ions when the Reynolds number is small as shown by Armaly et al.. ${ }^{15)}$ However, the length becomes small when the Reynolds number is larger than 1,000 when $\xi=1.5$. This is due to the flow structure change as described later.

Figure 8 shows there exists effective penetration when the molar ratio is set from 1.5 to 5.0 and the Reynolds number is low. When $\xi=1.5$, the flow reattachment always occurs in the present conditions. On the other hand, no reattachment is observed in all cases when $\xi=0.5$ and in higher Reynolds number range when $\xi=3.0$ and 5.0. When $\xi=0.5$ and 5.0, the second relaxation time is not observed and the first normal stress shows low values. Thus, the weak elasticity causes the weak penetration.

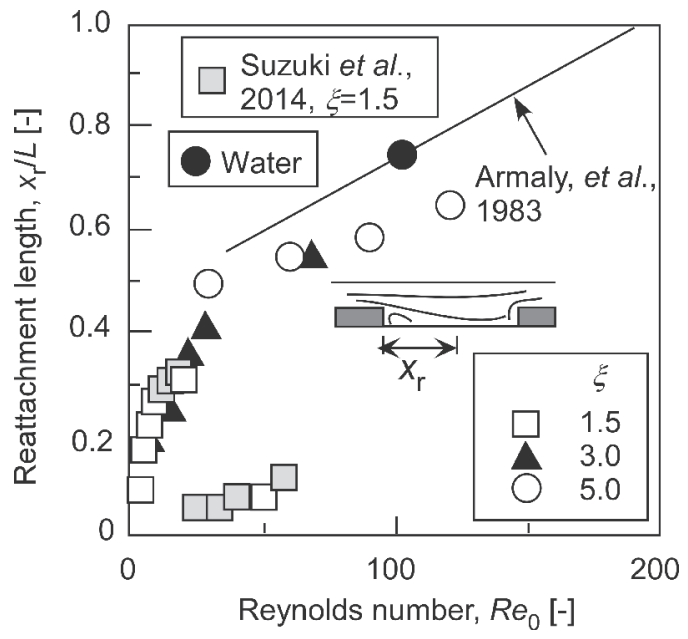

Fig. 10. Reattachment length on the zero-shear Reynolds number.
From Figure 9, the recirculating region size is observed to increase with decrease of the molar ratio of counter-ions from 5.0 to 1.5 . It is also found that the size linearly increases with the Reynolds number in the low Reynolds number region. This is due to increase of the Weissenberg number.

Figure 10 show a replot of reattachment length on zeroshear Reynolds number. The results on Newtonian fluid flow obtained by Armaly et al. ${ }^{15)}$ are also plotted. From the figure, it is found that all reattachment lengths exist on one curve except the results when $\xi=1.5$ and $R e>1,000$. The Barus effect occurs due to fluid elasticity in low Reynolds number region. However, the reattachment length is dominated by flow inertia in a high Reynolds number region. Thus, the reattachment length when $R e_{0}>50$ asymptotically approaches to the results reported by Armaly et al.. ${ }^{15)}$

On the separation region in the downstream of the cavity, Suzuki $^{6}$ theoretically revealed that the size can be a function of expansion ratio, $\beta[-](=W /(W-H))$ and the Weissenberg number as follows.

$$
\frac{x_{r}}{H} \propto 2 \frac{W e}{\beta}\left(\beta^{2}+\beta+1\right)
$$

Figure 11 shows a re-plot of the separation length on the function of $\beta$ and $W e$. In the figure, the experimental results in the previous study are shown as a solid line.

From this figure, the present results well agree with the previous results and the theory as shown with Eq. (5) when the Weissenberg number is low corresponding to the low Reynolds number region.

On the other hand, the recirculating region size takes a peak value as $\operatorname{Re}=1,000$ and decrease after the peak when

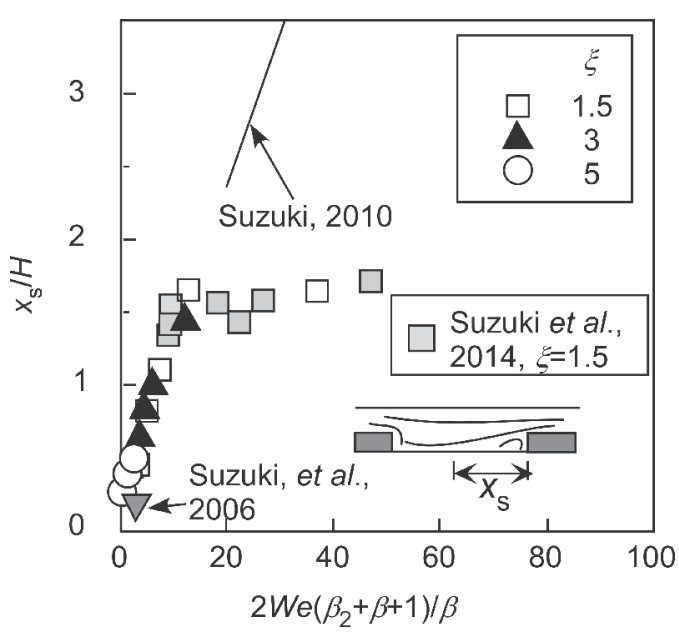

Fig. 11. Comparison of the separation length with theoretical prediction obtained by Suzuki, 2010 
$\xi=1.5$ as shown in Figure 9 and also in Figure 11. When the Reynolds number ranges from 1,300 to 2,500 , the flow separated from the top corner of the upstream rib protrudes toward the downstream rib. As the Weissenberg number is also large, however, the fluid flows backward and reattaches onto the bottom of the cavity, there, as shown in Figure 12. This bulge-like flow structure causes the small reattachment length observed in the upstream region of the cavity shown in Figure 7 and disturbs the formation of the recirculating region in the downstream region of the cavity shown in Figure 9 as discussed in the previous study ${ }^{16}$ As mentioned above, an open channel was used in the previous study ${ }^{6}$ and its aspect ratio was 5 . When the flow geometry was used, bulge-like flow motion was not observed. This structure appearance might relate to the aspect ratio or the pressure field. The previous study ${ }^{16)}$ also reported the bulge structure has highlevel fluctuation. This fluctuation causes the separation size fluctuation not only streamwisely but also spanwisely. In this stage, the three-dimensional fluctuations of this bulge structure are not clarified, but these flow fluctuations can be expected to contribute the heat transfer enhancement. On this, a further investigation is required.

From the above discussions, it is concluded that the effective flow penetration related to the heat transfer augmentation in the cavity can be obtained when the molar ratio was set from 1.5 to 5.0 and the Reynolds number is low.

\subsection{Pressure Coefficients}

Figure 13 shows the pressure coefficients, $C_{\mathrm{p}}[-]$, between the inlet and the outlet of the test section with $1,100 \mathrm{~mm}$ of the total length including five ribs and four cavities. The pressure coefficient is defined by use of the mean velocity in the narrow flow space as follows.

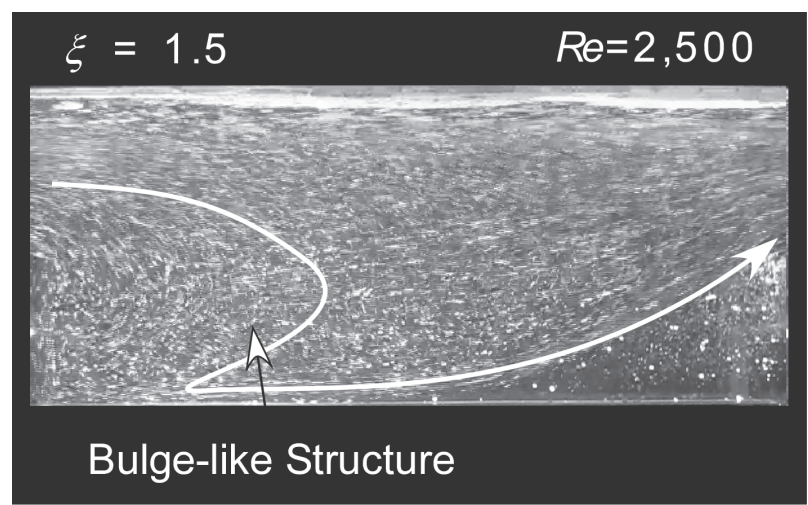

Fig. 12. Bulge-like flow structure observed in the previous study ${ }^{16)}$.

$$
C_{\mathrm{p}}=\frac{\Delta P}{\frac{1}{2} \rho U_{m}^{2}}
$$

Here, $\Delta P[\mathrm{~Pa}]$ is the total pressure loss through the test section.

Kunugi and Takizuka ${ }^{18)}$ reported the numerical results on the pressure loss when the fluid was Newtonian and the ribs mounted on the one-side wall. Their result for one pitch, $C_{\mathrm{p}}^{*}[-]$, is follows.

$$
C_{\mathrm{p}}^{*}=\frac{191.7}{R e^{*}}
$$

Here, $\operatorname{Re}^{*}[-]$ is the Reynolds number defined with the hydraulic diameter of the width of the wider flow path and with the mean velocity in the wider flow path. In their case, the length of the rib to the pitch, $R /(L+R)$, and the rib height to the width of the wider flow path, $H / W$, were set at $1 / 7$ and $1 / 3$, respectively, while those in the present case were set at $1 / 2$ and $1 / 2$, respectively. The solid line in the figure shows their results modified for the present case under the assumption that the pressure loss linearly increase with $R /(L+R)$ and to $H / W$. This assumption was also adopted in the previous numerical study ${ }^{19)}$ and was effective. In order to show the present validation on the pressure loss measurements, the results in the cases of water are also plotted in the figure.

From this figure, it is found that the pressure loss coefficient linearly decreases with the Reynolds number in each case. In the case of water, the values well agree with the results obtained by Kunugi and Takizuka. ${ }^{18)}$

When $\xi=1.5$, the pressure loss shows much higher than the results of other cases. This is due to the high viscosity and

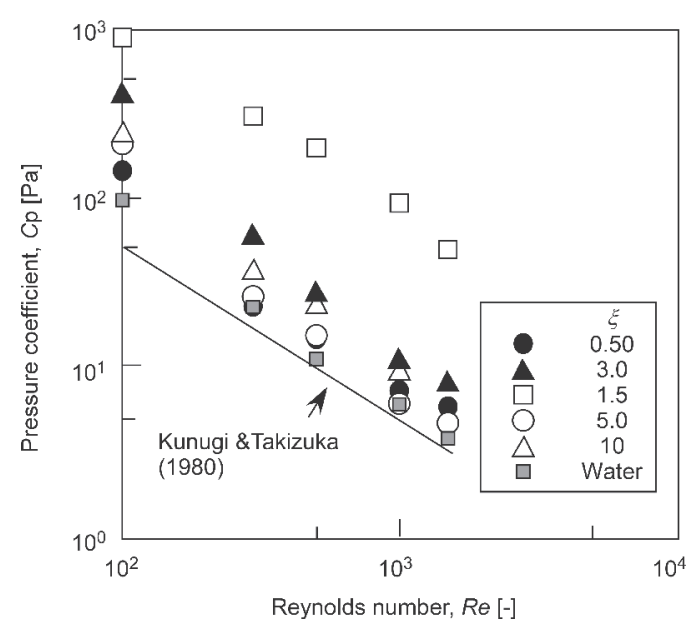

Fig. 13. Pressure coefficient on solvent Reynolds number. 
elasticity of the fluid and it relates to the strong penetration into the cavity. However, the results in the case when $\xi=5.0$ shows almost the same pressure loss as those of water, though the effective flow penetration into the cavity occurs in the low Reynolds number region. The flow penetration might cause the heat transfer augmentation from the cavity bottom. Thus, the present results on $\xi=5.0$ indicate that the heat transfer augmentation can be obtained without the penalty of pressure loss increase by some optimum fluid having suitable elasticity and low viscosity.

\section{CONCLUSIONS}

An experimental study has been performed in order to investigate the optimum condition on the heat transfer augmentation technique using a Barus effect of visco-elastic fluids. The rheological characteristics were changed by the change of the molar ratio of counter-ions to surfactants. The effect of the molar ratio of counter-ions on the flow behaviour was investigated by the flow visualization. The pressure loss measurement was also conducted in order to evaluate the effect of the molar ratio of counter-ions. From the results, the following conclusions were obtained:

1. The effective flow penetration into the cavity can be observed when the molar ratio of counter-ions is set from 1.5 to 5.0 and the Reynolds number is kept low.

2. The flow reattachment length formed by the flow separation from the top corner of the upstream rib and the flow reattachment onto the cavity bottom increases with the Reynolds number until $R e=400$ and with decrease of the molar ratio from 5.0 to 1.5 .

3. The length of the flow separation formed between the flow separated from the cavity bottom and the downstream rib increases with Reynolds number and with the molar ratio from 1.5 to 5.0 .

4. The pressure coefficient becomes large in the cases when the marked Barus effect can be observed. However, the pressure coefficients when $\xi=5.0$ shows almost the same values than those of water.

5. The optimum fluid for heat transfer augmentation and for low pressure-loss penalty is required to have high elasticity and low viscosity. Among the present cases, the results on 5.0 of the molar ratio of counter-ions show the effective flow penetration and pressure loss characteristics when the Reynolds number is set at less than 400 .

In the next stage, the heat transfer characteristics for the cases reported in the present study will be investigated.

\section{NOMENCLATURE}

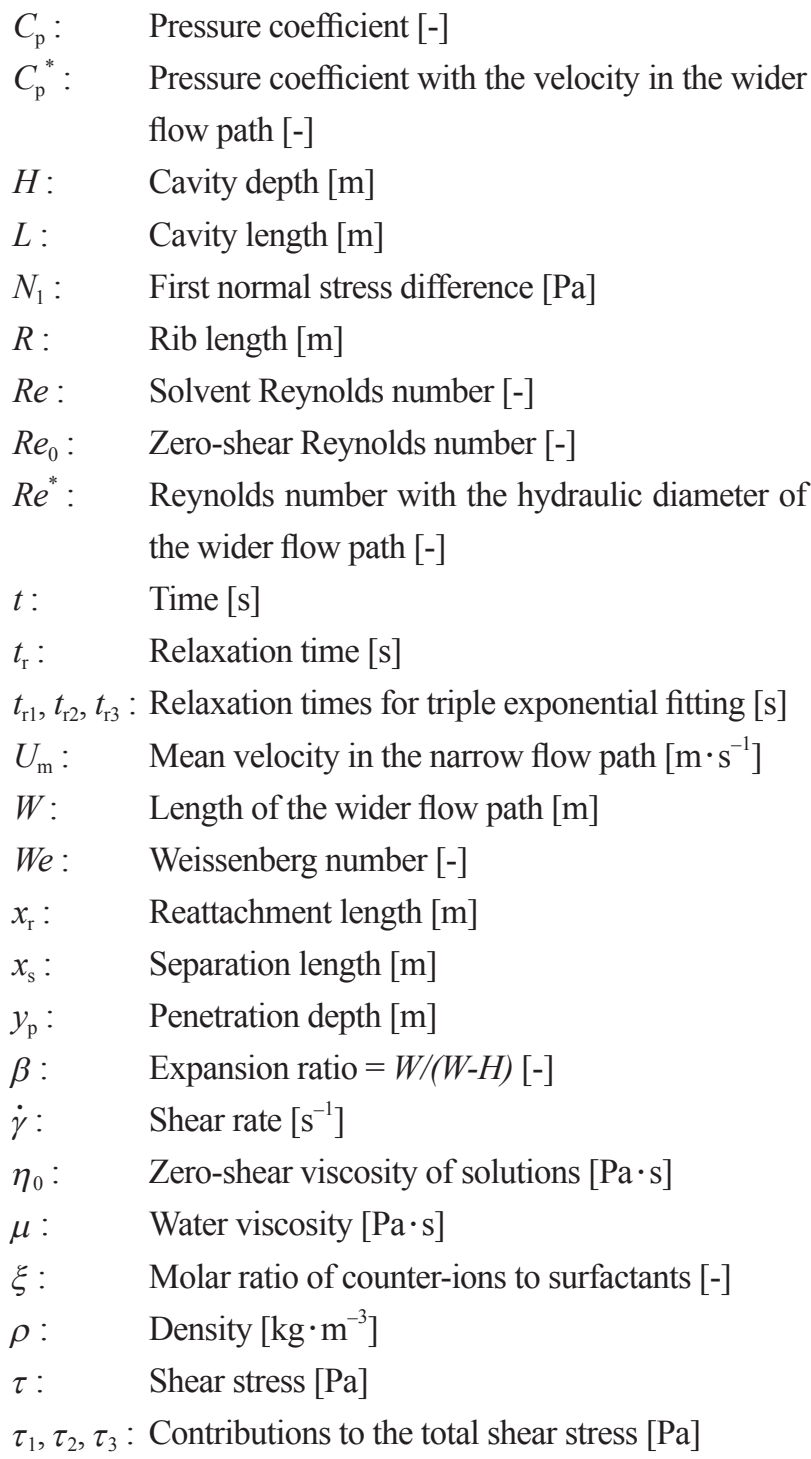

\section{REFERENCES}

1) Aung $W$, An Interferometric Investigation of Separated Forced Convection in Laminar Flow Past Cavities, Trans ASME, $J$ Heat Transfer, 105, 505-512 (1983).

2) Greiner N, An Experimental Investigation of Resonant Heat Transfer Enhancement in Grooved Channels, Int J Heat Mass Transfer, 34, 1383-1391 (1991).

3) Suzuki H, Kida S, Nakamae T, Suzuki K, Flow and Heat Transfer over a Backward-Facing Step with a Cylinder Mounted near Its Top Corner, J Heat Fluid Flow, 12, 353-359 (1991).

4) Nishimura $T$, Oka N, Yoshinaka $Y$, Kunitsugu K, Influence of Imposed Oscillatory Frequency on Mass Transfer Enhancement of Grooved Channels for Pulsatile Flow, Int $J$ Heat Mass Transfer, 43, 2365-2374 (2000).

5) Suzuki H, Ishiba H, Usui H, Heat Transfer Characteristics in a Cavity of a Symmetric Grooved Channel with Visco-Elastic Fluid, Proc $12^{\text {th }}$ International Heat Transfer Conference, 2, 177-182, Grenoble (2002). 
6) Suzuki H, Separation Characteristics of Visco-Elastic Fluid in a Cavity, J Chemical Engineering, Japan, 43, 421-428 (2010).

7) Suzuki H, Yamada S, Usui H, Numerical Computations on Heat Transfer Characteristics from the Cavity Bottom in Parallel Plates Swept by Viscoelastic Fluid. J Chemical Engineering, Japan, 39, 915-923 (2006).

8) Suzuki H, Yamada S, Usui H, Numerical Study on ViscoElastic Fluid Flow and Heat Transfer Characteristics in a Cavity, Proc $1^{\text {st }}$ Int Forum on Heat Transfer, 245-246, Kyoto (2004).

9) Mckinley GH, Raiford WP, Brown RA, Armstrong RC, Nonlinear Dynamics of Viscoelastic Flow in Axisymmetric Abrupt Contractions, J Fluid Mechanics, 223, 411-456 (1991).

10) Mongruel A, Cloitre M, Axisymmetric Orifice Flow for Measuring the Elongatoinal Viscosity of Semi-Rigid Polymer Solutions, J Non-Newtonian Fluid Mechanics, 110, 27-43 (2003).

11) Usui H, Kamada T, Suzuki H, Surfactant Drag Reduction Caused by a Cationic Surfactant with Excess Addition of Counter-Ion, J Chemical Engineering, Japan, 37, 1232-1237 (2004).

12) Lu B, Zheng Y, Davis HT, Scriven LE, Talmon Y, Zakin JL, Effect of Variation in Counterion to Surfactant Ratio on Rheology and Microstructures of Drag Reducing Cationic Surfactant Systems, Rheologica Acta, 37, 528-548 (1998).
13) Suzuki H, Higuchi Y, Watanabe H, Komoda Y, Ozawa S, Nishimura T, Takenaka N, Relaxation Behavior of a DragReducing Cationic Surfactant Solution, Nihon Reoroji Gakkaishi, 40, 85-90 (2012).

14) Zheng $Y$, Lin Z, Zakin JL, Cryo-TEM Imaging the FlowInduced Transition from Vesicle to Thread-like Micelles, $J$ Physical Chemistry, B, 104, 5263-5271 (2000).

15) Armaly BF, Durst F, Pereira JCF, Schoenung B, Experimental and Theoretical Investigation of Backward-Facing Step Flow, J Fluid Mechanics, 127, 473-496 (1983).

16) Suzuki H, Sato H, Hidema R, Komoda Y, Bulge Structure in a Cavity Swept by a Viscoelastic Fluid, J Physics: Conf Ser, 530, \#012055 (2014).

17) Chiang TP, Sheu TWH, A Numerical Revisit of BackwardFacing Step Flow Problem, Physics of Fluids, 11-4, 862-874 (1999).

18) Kunugi T, Takizuka T, Flow and Heat Transfer in Parallel Channel Attached with Equally-Spaced Ribs, 2nd Report, Japan Atomic Energy Research Institute M Reports, \#9115 (1980).

19) Suzuki H, Hidema R, Komoda Y, Size Effect of the Flow Path on the Flow and Heat Transfer Characteristics in a Cavity Swept by a Visco-Elastic Fluid, International Heat Transfer Conference 15, DOI: 10.1615/IHTC15.hex.009449, Begell House inc., New York (2014). 